\title{
SMALL BALL PROBABILITIES FOR STABLE CONVOLUTIONS
}

\author{
Frank Aurzada ${ }^{1}$ And Thomas Simon ${ }^{2}$
}

\begin{abstract}
We investigate the small deviations under various norms for stable processes defined by the convolution of a smooth function $f:] 0,+\infty[\rightarrow \mathbb{R}$ with a real $S \alpha S$ Lévy process. We show that the small ball exponent is uniquely determined by the norm and by the behaviour of $f$ at zero, which extends the results of Lifshits and Simon, Ann. Inst. H. Poincaré Probab. Statist. 41 (2005) 725-752 where this was proved for $f$ being a power function (Riemann-Liouville processes). In the Gaussian case, the same generality as Lifshits and Simon, Ann. Inst. H. Poincaré Probab. Statist. 41 (2005) 725-752 is obtained with respect to the norms, thanks to a weak decorrelation inequality due to Li, Elec. Comm. Probab. 4 (1999) 111-118. In the more difficult non-Gaussian case, we use a different method relying on comparison of entropy numbers and restrict ourselves to Hölder and $L_{p}$-norms.
\end{abstract}

Mathematics Subject Classification. 60F99, 60G15, 60G20, 60G52.

Received April 4, 2006. Revised July 10, 2006.

\section{INTRODUCTION}

Small ball probability problems consist in finding the right asymptotics of the function

$$
\log \mathbb{P}[\|X\|<\varepsilon]
$$

when $\varepsilon \rightarrow 0$ for a given process $X$ and norm $\|$.$\| , with an expected behaviour in -K \varepsilon^{-\gamma}$ for some constants $\gamma, K>0$. A particular feature of these large deviation type estimates is that the critical exponent $\gamma$ usually depends, among other parameters, on the regularity of the process $X$, which is not the case in classical large deviations where $\gamma$ enjoys more universality. More precisely, a general rule is that a smoother process will have more chance to stay close to the zero function, so that $\gamma$ will be smaller. For example, one has [13]

$$
\varepsilon^{1 / H} \log \mathbb{P}\left[\sup _{t \in[0,1]}\left|B_{t}^{H}\right|<\varepsilon\right] \rightarrow-\mathscr{K} \in(0,+\infty), \quad \varepsilon \rightarrow 0,
$$

Keywords and phrases. Entropy numbers, fractional Ornstein-Uhlenbeck processes, Riemann-Liouville processes, small ball probabilities, stochastic convolutions, wavelets.

1 Institut für Mathematik, Technische Universität Berlin, Straße des 17. Juni 136, 10623 Berlin, Germany; aurzada@math.tu-berlin.de

2 Equipe d'analyse et probabilités, Université d'Evry-Val d'Essonne, boulevard François Mitterrand, 91025 Evry Cedex, France; tsimon@univ-evry.fr 
where $B^{H}$ stands for the fractional Brownian motion with Hurst parameter $H$. Consider now a more general stable Volterra process defined by

$$
M_{t}=\int_{\mathbb{R}} K(t, x) Z(\mathrm{~d} x)
$$

where $Z$ is a stable random measure and $K: \mathbb{R}^{2} \rightarrow \mathbb{R}$ some integration kernel. Several examples [19] show that the sample path regularity of $M$ is determined by the behaviour of $K$ on the diagonal, so that one should try to connect this behaviour with the small ball probabilities of $M$. In this paper, we investigate this connection within the framework of convolution kernels: we study processes of the type

$$
X_{t}=\int_{0}^{t} f(t-s) \mathrm{d} Z_{s}, \quad t \in[0,1],
$$

where $Z$ is a real symmetric $\alpha$-stable Lévy process $(\alpha \in(0,2])$ and $f:[0,1] \rightarrow \mathbb{R}$ is a function which is smooth, except possibly at zero. When $\alpha<1$, the integral in (1.1) is understood in the Riemann-Stieltjes sense, since $Z$ has bounded variation. When $Z$ has unbounded variation that is $\alpha \geq 1$, the integral in (1.1) is understood in the stochastic sense. Integrating by parts, it is easy to see that the regularity of $X$ depends only on the behaviour of $f$ at 0 . For example, if $f^{(0)}(0)=\ldots=f^{(k-1)}(0)=0$ and $f^{(k)}(0) \neq 0$ for some $k \geq 0$, then

$$
X_{t}=f^{(k)}(0) A_{t}^{k}+\int_{0}^{t} f^{(k+1)}(t-u) A_{u}^{k} \mathrm{~d} u, \quad t \in[0,1],
$$

where

$$
A_{t}^{0}=Z_{t} \quad \text { and } \quad A_{t}^{i+1}=\int_{0}^{t} A_{s}^{i} \mathrm{~d} s
$$

for every $i \geq 0$, so that $X$ has the same regularity as $A^{k}$. In the homogeneous case $f(x) \equiv x^{c}$ for some $c \geq 0$, the process $X$ is the $\alpha$-stable Riemann-Liouville (or fractional integrated) process $R^{H}$ with self-similarity parameter $H=c+1 / \alpha>0$ :

$$
R_{t}^{H}=\int_{0}^{t}(t-s)^{H-1 / \alpha} \mathrm{d} Z_{s}, \quad t \in[0,1],
$$

a process whose small deviations were recently studied under a wide class of semi-norms [16]. Assuming now that $f(x) \equiv x^{c} g(x)$ for some $c \geq 0$ and $g$ is, say, an analytic function in the neighbourhood of zero, we can represent $X$ as a mixture of non-independent RL processes:

$$
X_{t}=\sum_{n=0}^{\infty} a_{n} R_{t}^{H+n}, \quad t \in[0,1],
$$

where $\left\{a_{n}, n \geq 0\right\}$ is a sequence of real numbers with $a_{0}=1$. The processes $X$ and $R^{H}$ have the same regularity, and the purpose in this paper is to show that they have also the same small ball probabilities.

In Section 2, the Gaussian case $\alpha=2$ is investigated. Borrowing wavelet techniques from [16] and using a corollary to a weak decorrelation inequality due to Li [11], we can separate both terms $R^{H}$ and $X-R^{H}$, and show the desired result, together with the existence of the small deviation constant for $X$. Recall indeed that Li's decorrelation inequality states that

$$
\gamma(A \cap B) \geq \gamma(\lambda A) \gamma\left(\left(1-\lambda^{2}\right)^{1 / 2} B\right)
$$

for every $\lambda \in(0,1)$, where $\gamma$ is a centered Gaussian measure on a Banach space $E$ and $A, B$ two symmetric convex sets in $E$. Letting $\lambda$ tend to 0 or 1 in (1.3) makes it then possible to preserve the rate and the constant in Gaussian small deviation problems - see Theorem 1.2 in [11]. From the generality of (1.3), one should expect our separation technique to be efficient for more general Gaussian Volterra processes as well, but we did not search in this direction further. 
In the non-Gaussian case, no decorrelation inequality like (1.3) is available for stable measures and we have to employ other tools, which lead unfortunately to less general results with respect to the norms. The results are also less precise since we cannot prove the existence of the small ball constant. The latter problem is probably difficult without Gaussianity, and it is not completely clear either if the constant really exists in this non-self-similar context.

In Section 3, we study the small deviations of $X$ under $L_{p}$ and Hölder norms in the non-Gaussian case. Whereas the lower bound comes rather easily after a time-integration formula, the upper bounds turns out to be more involved without self-similarity, and we have to use the connection between small deviation and metric entropy for stable processes which was discovered in [15]. It is more than likely that other norms could be tackled along the same way, but an annoying feature of entropy numbers is their dependence on dual function spaces which become increasingly complicated, so that it seems difficult to use them within a global formalism, as in [16]. We also feel that these two particular cases have some generality: the critical exponent does not depend on the norm in the first case, and does in the second.

In Section 4, we discuss two important examples: the stable Ornstein-Uhlenbeck processes (which was the starting point of this research) where $f(x)=\mathrm{e}^{x}$ in the representation (1.1), and the fractional OrnsteinUhlenbeck processes as recently introduced in $[5,17,21]$, where $f(x)=x^{c} \mathrm{e}^{x}$ in the representation (1.1). These new fractional processes seem important for applications, especially in network traffic [21]. Let us finally mention that convolution processes like (1.1) have been studied in mathematical finance for the so-called forward interest rate curve [8].

Throughout, we will denote by $C$ a constant independent of the involved parameters whose value may change from line to line, and we will use the functional notation $f(x) \approx g(x)$ if there are constants $C_{1}, C_{2}$ such that $0<C_{1}<f(x) / g(x)<C_{2}<+\infty$ for all sufficiently small or large $x$. Unless explicitly mentioned, every function or process will be considered on $[0,1]$ only and starting from 0 .

\section{The Gaussian CASE}

Let us first briefly recall the definition of the set $\mathbf{N}(\beta, p)$ of $\beta$-self similar and $p$-pseudo additive semi-norms - in abbreviation $(\beta, p)$ semi-norms, which was introduced in [16]. If $\mathscr{F}$ is a linear space of real functions containing the set $\mathscr{C}_{K}^{\ell}$ (functions having a continuous derivative of $l$-th order and compact support) for some $\ell \in \mathbb{N}$, a semi-norm $\|\cdot\|$ on $\mathscr{F}$ belongs to $\mathbf{N}(\beta, p)$ if the following properties hold:

(A) $\|\cdot\|_{I} \leq\|\cdot\|_{J}$ for all real intervals $I, J$ such that $I \subseteq J$.

(B) For every interval $I$ and $f, f_{n} \in \mathscr{F}$, if $f_{n} \rightarrow f$ uniformly on $I$ then $\|f\|_{I} \leq \lim \sup \left\|f_{n}\right\|_{I}$.

(C) $\|f\|_{I-c}=\|f(\cdot-c)\|_{I}$ for every interval $I, f \in \mathscr{F}$, and $c \in \mathbb{R}$.

(D) $\|f(c \cdot)\|_{I / c}=c^{\beta}\|f\|_{I}$ for every interval $I, f \in \mathscr{F}$, and $c>0$.

(E) For every $a_{0}<\ldots<a_{n} \in \mathbb{R}$ and $f \in \mathscr{F}$

$$
\begin{cases}\|f\|_{\left[a_{0}, a_{n}\right]} \geq\left(\|f\|_{\left[a_{0}, a_{1}\right]}^{p}+\cdots+\|f\|_{\left[a_{n-1}, a_{n}\right]}^{p}\right)^{1 / p} & \text { if } p<+\infty \\ \|f\|_{\left[a_{0}, a_{n}\right]} \geq \sup \left(\|f\|_{\left[a_{0}, a_{1}\right]}, \ldots,\|f\|_{\left[a_{n-1}, a_{n}\right]}\right) & \text { if } p=+\infty .\end{cases}
$$

(F) There exists $C_{p}>0$ such that for every $a_{0}<\ldots<a_{n} \in \mathbb{R}$ and $f \in \mathscr{C}_{K}^{\ell}$ verifying $f\left(a_{0}\right)=\cdots=$ $f\left(a_{n}\right)=0$,

$$
\begin{cases}\|f\|_{\left[a_{0}, a_{n}\right]} \leq C_{p}\left(\|f\|_{\left[a_{0}, a_{1}\right]}^{p}+\cdots+\|f\|_{\left[a_{n-1}, a_{n}\right]}^{p}\right)^{1 / p} & \text { if } p<+\infty, \\ \|f\|_{\left[a_{0}, a_{n}\right]} \leq C_{\infty} \sup \left(\|f\|_{\left[a_{0}, a_{1}\right]}, \ldots,\|f\|_{\left[a_{n-1}, a_{n}\right]}\right) & \text { if } p=+\infty .\end{cases}
$$


Notice that in this definition the compatibility properties (A-B) are quite innocuous and always satisfied in practice. More is demanded in properties (C-F) which mean respectively translation-invariance, $\beta$-self-similarity, $p$-subadditivity and $p$-superadditivity of the semi-norm. In [16] it is observed that all classical semi-norms fall into $\mathbf{N}(\beta, p)$ 's scope, except perhaps Solovietsky and Besov norms for which one needs the weaker notion of $(\beta, p)$-semi-norm in the wide sense - i.e. with respect to some wavelet basis - see [16] for more details. In that paper, it was also remarked that the inequality $\beta+1 / p \geq 0$ is verified by all classical examples, an interesting fact for which no sensible explanation was given as yet.

The main result of [16] entails that if $\|$.$\| is a (\beta, p)$-semi-norm and $X \equiv R^{c+1 / 2}$ is defined by (1.1) with $\alpha=2$ and $f(x)=x^{c}$ for $c>\beta+1 / p-1 / 2$, there exists $\mathscr{K} \in(0,+\infty)$ such that

$$
\lim _{\varepsilon \downarrow 0} \varepsilon^{\gamma} \log \mathbb{P}\left[\left\|R^{c+1 / 2}\right\| \leq \varepsilon\right]=-\mathscr{K}
$$

with $\gamma=(c+1 / 2-\beta-1 / p)^{-1}$. This formula for the rate synthezises all cases previously studied for the small balls of RL processes. In this section, our purpose is to prove that the result also holds for more general moving average Gaussian processes. In the following we will denote by $\operatorname{Ent}(x)$ the integer part of a real number $x$ and $\mathscr{C}^{i}$ will stand for the set of functions from $[0,1]$ to $\mathbb{R}$ with continuous derivative of $i$-th order.

Theorem 2.1. Let $X$ be defined in (1.1) such that $\alpha=2$ and $f(x)=x^{c} g(x)$ for $c>-1 / 2$ and $g:[0,1] \rightarrow \mathbb{R}$ with $g(0)=1$. Suppose that $g$ belongs to $\mathscr{C}^{k+1}$ with $k=\operatorname{Ent}(c+1)$. For any seminorm $\|\cdot\| \in \mathbf{N}(\beta, p)$ with $c+1 / 2>\beta+1 / p$, we have

$$
\lim _{\varepsilon \downarrow 0} \varepsilon^{\gamma} \log \mathbb{P}[\|X\| \leq \varepsilon]=-\mathscr{K}
$$

where $\gamma=(c+1 / 2-\beta-1 / p)^{-1}$ and $\mathscr{K}$ is given by $(2.1)$.

Proof. Set $H=c+1 / 2>0$ and consider the difference process $X^{H}=X-R^{H}$. Integrating by parts, we can write

$$
X_{t}^{H}=h^{(k)}(0) A_{t}^{k}+\int_{0}^{t} h^{(k+1)}(t-u) A_{u}^{k} \mathrm{~d} u, \quad t \geq 0
$$

with the notation of the introduction and where we set $h(x)=x^{c}(g(x)-1)=O\left(x^{c+1}\right)$ in the neighbourhood of zero. By (2.1) and Theorem 1.2 in [11], it suffices to show that

$$
\lim _{\varepsilon \downarrow 0} \varepsilon^{\gamma} \log \mathbb{P}\left[\left\|X^{H}\right\| \leq \varepsilon\right]=0,
$$

and we will actually prove a little more:

$$
\liminf _{\varepsilon \downarrow 0} \varepsilon^{\gamma^{\prime}} \log \mathbb{P}\left[\left\|X^{H}\right\| \leq \varepsilon\right]>-\infty,
$$

with $\gamma^{\prime}=(k+1 / 2-\beta-1 / p)^{-1}<\gamma$ since $k=\operatorname{Ent}(c+1)>c$. This will be done in the same way as Theorem 7 in [16] and we will also use the same notation. Suppose first that $c$ is not an integer and that $p<+\infty$. Notice that $h^{(k)}(0)=0$ in (2.2). Setting $\ell=k+1>k+1 / 2=k^{\prime}$ and decomposing $X^{H}$ along the wavelet basis $\left\{\psi_{j n}, n \leq 2^{j}\right\}$ defined in [16] p. 736, we get

$$
\left\|X^{H}\right\| \leq C \sum_{j \geq 0}\left(\sum_{n \leq 2^{j}}\left|r_{j n}\right|^{p}|| \psi_{j n} \|^{p}\right)^{1 / p} \leq C \sum_{j \geq 0} 2^{(1 / 2+\beta) j}\left(\sum_{n \leq 2^{j}}\left|r_{j n}\right|^{p}\right)^{1 / p}
$$


where $C$ is some constant independent of $j, n$ and

$$
\begin{aligned}
r_{j n} & =2^{-j / 2} \int_{0}^{+\infty} X^{H}\left(2^{-j} s\right) \psi(s-n) \mathrm{d} s \\
& =2^{-\left(k^{\prime}+1 / 2\right) j} \int_{0}^{+\infty} 2^{k^{\prime} j} X^{H}\left(2^{-j} s\right) \psi(s-n) \mathrm{d} s \\
& =2^{-\left(k^{\prime}+1 / 2\right) j} \int_{0}^{+\infty} 2^{k^{\prime} j}\left(\int_{0}^{2^{-j} s} h^{(k+1)}\left(2^{-j} s-u\right) A_{u}^{k} \mathrm{~d} u\right) \psi(s-n) \mathrm{d} s \\
& \underline{\underline{d}} 2^{-\left(k^{\prime}+3 / 2\right) j} \int_{0}^{+\infty}\left(\int_{0}^{s} h^{(k+1)}\left(2^{-j}(s-u)\right) A_{u}^{k} \mathrm{~d} u\right) \psi(s-n) \mathrm{d} s \\
& =2^{-\left(k^{\prime}+3 / 2\right) j} \int_{\mathbb{R}}^{s+n} \psi(s)\left(\int_{0}^{s+1)} h^{(k+1)}\left(2^{-j}(s+n-u)\right) A_{u}^{k} \mathrm{~d} u\right) \mathrm{d} s,
\end{aligned}
$$

the equality in law coming from the $(k+1 / 2)$-self-similarity of $A^{k}$. This finally entails

$$
\left\|X^{H}\right\| \leq C \sum_{j \geq 0} 2^{-\left(k^{\prime}-\beta-1 / p\right) j}\left(\sup _{|n| \leq M 2^{j}}\left|r_{j n}^{\prime}\right|\right)
$$

with the notation

$$
r_{0 n}^{\prime}=r_{0 n} \quad \text { and } \quad r_{j n}^{\prime} \stackrel{d}{=} 2^{-j} \int_{\mathbb{R}} \psi(s)\left(\int_{0}^{s+n} h^{(k+1)}\left(2^{-j}(s+n-u)\right) A_{u}^{k} \mathrm{~d} u\right) \mathrm{d} s \text { if } j \geq 1 .
$$

We can rewrite the wavelet coefficients $r_{j n}^{\prime}=\tau_{j n} y_{j n}$ with

$$
\tau_{j n}=2^{-j}\left(\int_{0}^{n+D}\left|\int_{-D}^{D} \psi(t) \mathrm{d} t \int_{0}^{t+n} h^{(k+1)}\left(2^{-j} v\right)(t+n-v-s)_{+}^{k} \mathrm{~d} v\right|^{2} \mathrm{~d} s\right)^{1 / 2}
$$

and $\left\{y_{j n},|n| \leq M 2^{j}, j \geq 0\right\}$ is an array of identically distributed jointly Gaussian random variables. As in Theorem 7 of $[16]$, it suffices to prove

$$
\sup _{n, j} \tau_{j n}^{2}<+\infty
$$

We have

$$
\tau_{j n}^{2}=4^{-j} \int_{0}^{n+D}\left|\int_{-D}^{D} \psi(t) \mathrm{d} t \int_{0}^{t+n} h^{(k+1)}\left(2^{-j} v\right)(t+n-v-s)_{+}^{k} \mathrm{~d} v\right|^{2} \mathrm{~d} s
$$

and we transform

$$
\int_{-D}^{D} \psi(t) \mathrm{d} t \int_{0}^{t+n} h^{(k+1)}\left(2^{-j} v\right)(t+n-v-s)_{+}^{k} \mathrm{~d} v
$$

through $\ell=k+1$ successive integrations by parts. The $\ell$-th derivative of

$$
t \mapsto \int_{0}^{t+n} h^{(k+1)}\left(2^{-j} v\right)(t+n-v-s)_{+}^{k} \mathrm{~d} v=\int_{0}^{t+n-s} h^{(k+1)}\left(2^{-j} v\right)(t+n-v-s)^{k} \mathrm{~d} v
$$

is given by

$$
t \mapsto k ! h^{(k+1)}\left(2^{-j}(t+n-s)\right)
$$


so that finally

$$
\tau_{j n}^{2}=C 4^{-j} \int_{0}^{n+D}\left|\int_{-D}^{D} I^{\ell} \psi(t) h^{(k+1)}\left(2^{-j}(t+n-s)\right) \mathbf{1}_{\{s \leq t+n\}} \mathrm{d} t\right|^{2} \mathrm{~d} s
$$

We cut the domain of integration in $s$ into $[0, n-D-1]$ and $[n-D-1, n+D]$. On the first interval it is easily seen by the smoothness of $g$ that the integrated function is bounded, so that after a change of variable we obtain

$$
\begin{aligned}
\tau_{j n}^{2} & \leq C 4^{-j}\left(n+\int_{-D-1}^{D}\left|\int_{s}^{D} h^{(k+1)}\left(2^{-j}(t-s)\right) \mathrm{d} t\right|^{2} \mathrm{~d} s\right) \\
& \leq C 4^{-j}\left(n+4^{j}\left|\int_{0}^{2^{-j}(2 D+1)} h^{(k+1)}(u) \mathrm{d} u\right|^{2}\right) \\
& =C 4^{-j}\left(n+C 4^{j}\right) \leq C
\end{aligned}
$$

independently of $n, j$, where the third inequality comes from the definition of $h$ and the fact that $k \leq c+1$. This proves (2.4) and completes the proof in the case when $c$ is not an integer and $p<+\infty$. If $c$ is an integer, then $h^{(k)}(0)=(k-1) ! g^{\prime}(0)$ is not necessarily zero and we have a slightly more complicated wavelet decomposition for $X^{H}$. Using again the $(k+1 / 2)$-self-similarity of $A^{k}$ and reasoning as above we obtain

$$
\left\|X^{H}\right\| \leq C \sum_{j \geq 0} 2^{-\left(k^{\prime}-\beta-1 / p\right) j}\left(\sup _{|n| \leq M 2^{j}}\left|s_{j n}\right|\right)
$$

with $s_{0 n}^{\prime}=r_{0 n}$ and

$$
s_{j n} \stackrel{d}{=} \int_{\mathbb{R}} \psi(s)\left(2^{-j} \int_{0}^{s+n} h^{(k+1)}\left(2^{-j}(s+n-u)\right) A_{u}^{k} \mathrm{~d} u+A_{s+n}^{k}\right) \mathrm{d} s
$$

if $j \geq 1$. Analogously, we need an upper bound on

$$
\begin{aligned}
\sigma_{j n}^{2} & =\int_{0}^{n+D}\left|\int_{-D}^{D} \psi(t) \mathrm{d} t\left((t+n-s)_{+}^{k}+2^{-j} \int_{0}^{t+n} h^{(k+1)}\left(2^{-j} v\right)(t+n-v-s)_{+}^{k} \mathrm{~d} v\right)\right|^{2} \mathrm{~d} s \\
& \leq 2\left(\int_{0}^{n+D}\left|\int_{-D}^{D} \psi(t)(t+n-s)_{+}^{k} \mathrm{~d} t\right|^{2} \mathrm{~d} s+\tau_{j n}^{2}\right) \leq C
\end{aligned}
$$

independent of $j, n$ from the above computations and the proof of Theorem 7 in [16]. This finishes the proof in the case $p<+\infty$. Finally, the case $p=+\infty$ can be handled exactly in the same way in writing

$$
\left\|X^{H}\right\| \leq C \sum_{j \geq 0}\left(\sup _{n \in N_{j}}\left|r_{j n}\right|\left\|\psi_{j n}\right\|\right)
$$

and we leave the details to the reader. 


\section{The NOn-GaUssian CASE}

\subsection{Preliminaries and statement of the main result}

In this section, we investigate small deviations of processes defined by (1.1) such that $0<\alpha<2$ and $f(x)=x^{c} g(x)$ for some $c \geq 0$ and $g$ analytic with $g(0)=1$. Of course, one should wish to obtain the same level of generality as above, and actually a stable extension of Theorem 2.1 would be readily obtained if one could prove the analogue of Theorem 1.2 in [11] for stable measures. Unfortunately, the latter relies on the weak Gaussian decorrelation inequality (1.3), for which no stable version is available because the subordination argument does not apply to a product of probabilities. We do not know either whether it is possible to prove a stable version of Theorem 1.2 in [11] without the decorrelation inequality (1.3).

Our assumptions on $X$ will be more stringent than in the Gaussian case. Firstly, setting $H=c+1 / \alpha$, we will suppose that $H \geq 1 / \alpha$. This assumption is natural because it is in accordance with the sample path properties of $X$ : if $H<1 / \alpha$ it is known - see Theorem 10.2.3 in [19] - that $X$ is almost surely unbounded on [0,1], which makes the small ball problem irrelevant for all classical norms except possibly $L_{p}$-norms - see Section 6 in [16] for more details and comments. Secondly, we suppose that $g: \mathbb{R} \rightarrow \mathbb{R}$ is analytic in the neighbourhood of 0 with convergence radius $\rho \geq 1$. This entails

$$
g(s)=\sum_{n=0}^{\infty} \frac{g^{(n)}(0)}{n !} s^{n}, \quad s \in[0,1]
$$

and by Theorem 11.4.1 in [19] we can write

$$
X_{t}=\sum_{n=0}^{\infty} a_{n} R_{t}^{H+n}
$$

pointwise for all $t \in[0,1]$, with

$$
a_{n}=\frac{g^{(n)}(0)}{n !} \text { and } R_{t}^{H+n}=\int_{0}^{t}(t-s)^{H-1 / \alpha+n} \mathrm{~d} Z_{s}
$$

Notice that the RL processes $R^{H+n}$ in (3.1) integrate the same process $Z$ and hence are not independent. From now on, we will only consider processes written in the form (3.1) with the same underlying process $Z$ and an absolutely converging series $\left\{a_{n}, n \geq 0\right\}$. As mentioned in the introduction, we will focus on two families of norms only:

(a) The $L_{p}$-norms, $1 \leq p \leq+\infty$, defined as usual by

$$
\|f\|_{p}=\left(\int_{0}^{1}|f(s)|^{p} \mathrm{~d} s\right)^{1 / p} \text { for } p<+\infty \text { and }\|f\|_{\infty}=\sup _{s \in[0,1]}|f(s)| \text { for } p=+\infty
$$

(b) The $\eta$-Hölder norms, $0<\eta<1$, defined by

$$
\|f\|_{\eta}=\sup _{0 \leq s<t \leq 1} \frac{|f(t)-f(s)|}{|t-s|^{\eta}}
$$

on functions $f:[0,1] \rightarrow \mathbb{R}$ starting from 0 . 
Our main result is the following:

Theorem 3.1. Let $X$ be defined by (1.2) with $0<\alpha<2, H \geq 1 / \alpha$, and such that

$$
\sum_{n=1}^{\infty}\left|a_{n}\right| n^{H-1 / \alpha+\delta}<+\infty
$$

for some $\delta>0$. Then for every $1 \leq p \leq+\infty$

$$
\log \mathbb{P}\left(\|X\|_{p} \leq \varepsilon\right) \approx-\varepsilon^{-1 / H}, \quad \varepsilon \rightarrow 0 .
$$

Besides, for every $\eta \in(0,1)$, then

$$
\log \mathbb{P}\left(\|X\|_{\eta} \leq \varepsilon\right)=-\varepsilon^{-1 /(H-\eta)+o(1)}, \quad \varepsilon \rightarrow 0
$$

as soon as $H>1 / \alpha+\eta$.

\section{Remarks 3.2.}

(a) For the Hölder norms, the term $o(1)$ only appears in estimating the upper bound for the small deviation probability and the lower bound provides the exact rate under the exponential scale.

(b) The lower bound holds for both norms under the condition

$$
\sum_{n=0}^{\infty}\left|a_{n}\right| n^{H-1 / \alpha}<+\infty
$$

which is slightly weaker than (3.2). We believe that this latter condition is actually necessary for the wellposedness of our small ball problem - see Remark 3.6 thereafter. Notice that both conditions (3.3) and (3.2) are satisfied as soon as $g$ has a convergence radius $\rho>1$.

(c) For the Hölder norm, the additional assumption on $H$ is natural, except possibly for the relevant boundary case $H=1 / \alpha+\eta$, since it is plausible, as in [20], that $X$ has finite Hölder norm if and only if $H \geq 1 / \alpha+\eta$. One probably needs an entirely different method to tackle the situation $H=1 / \alpha+\eta$, this even for RL processes, since the wavelet arguments of [16] fail. Typically, one should expect some superexponential behaviour.

The proof of Theorem 3.1 will be given in two parts. First, the lower bound will be rather easily established using a time-integration lemma. For the more involved upper bound, we will need estimates for the entropy numbers of the underlying Riemann-Liouville operators, in order to apply then Li-Linde's upper criterion for general symmetric stable processes [14].

\subsection{Proof of the lower bound}

We begin with an easy lemma which states roughly that Riemann-Liouville processes form a semi-group w.r.t. time integration. Recall the notation

$$
\mathrm{B}(p, q)=\frac{\Gamma(p) \Gamma(q)}{\Gamma(p+q)}, \quad p, q>0
$$

for Euler's Beta function.

Lemma 3.3. For every $H \geq 1 / \alpha$ and $\beta>0$, we have a.s.

$$
\mathrm{B}(\beta, H-1 / \alpha+1) R_{t}^{H+\beta}=\int_{0}^{t}(t-s)^{\beta-1} R_{s}^{H} \mathrm{~d} s .
$$


Proof. Notice first that the integration of the right-hand side makes sense since almost surely $R^{H} \in L_{\infty}[0,1]$ and $s \mapsto(t-s)^{\beta-1} \mathbf{1}_{\{s \leq t\}}$ is in $L_{1}[0,1]$. We have a.s.

$$
\begin{aligned}
\int_{0}^{t}(t-s)^{\beta-1} R_{s}^{H} \mathrm{~d} s & =\int_{0}^{1} \mathbf{1}_{\{s \leq t\}}(t-s)^{\beta-1} \int_{0}^{1} \mathbf{1}_{\{u \leq s\}}(s-u)^{H-1 / \alpha} \mathrm{d} Z_{u} \mathrm{~d} s \\
& =\int_{0}^{t}\left(\int_{u}^{t}(t-s)^{\beta-1}(s-u)^{H-1 / \alpha} \mathrm{d} s\right) \mathrm{d} Z_{u}
\end{aligned}
$$

where in the second line we applied Fubini's theorem. Making the substitution $r=(s-x) /(t-x)$, we obtain

$$
\begin{aligned}
\int_{0}^{t}\left(\int_{u}^{t}(t-s)^{\beta-1}(s-u)^{H-1 / \alpha} \mathrm{d} s\right) \mathrm{d} Z_{u} & =\int_{0}^{t} \int_{0}^{1}(1-r)^{\beta-1} r^{H-1 / \alpha} \mathrm{d} r(t-u)^{\beta+H-1 / \alpha} \mathrm{d} Z_{u} \\
& =\mathrm{B}(\beta, H-1 / \alpha+1) \int_{0}^{t}(t-u)^{\beta+H-1 / \alpha} \mathrm{d} Z_{u}
\end{aligned}
$$

which completes the proof.

Let us now recall the definition of the Riemann-Liouville operator, which will also be useful in proving the upper bound:

Definition 3.4. The Riemann-Liouville operator with parameter $\beta>0$ is defined by

$$
\mathscr{R}_{\beta} y(t)=\frac{1}{\Gamma(\beta)} \int_{0}^{t}(t-s)^{\beta-1} y(s) \mathrm{d} s,
$$

as soon as the term on the right-hand side makes sense.

This operator has been extensively studied over the years in many areas of analysis and probability, see [7] and [18] for modern accounts. Our Lemma 3.3 entails that a.s.

$$
R^{H+\beta}=\frac{\Gamma(H+\beta-1 / \alpha+1)}{\Gamma(H-1 / \alpha+1)} \mathscr{R}_{\beta}\left(R^{H}\right) .
$$

Notice that $\mathscr{R}_{\beta}$ is a fractional integration operator, which is hence regularizing. In particular, given a function space $\mathscr{F}$ one should expect that $R^{H+n} \in \mathscr{F}$ for all $n \geq 0$ as soon as $R^{H} \in \mathscr{F}$, and it is natural to ask if the problem of comparing the small deviations of $R^{H}$ and $X$ is well-posed as soon as $R^{H} \in \mathscr{F}$ holds, in other words if

$$
\left\|R^{H}\right\|_{\mathscr{F}}<+\infty \Longrightarrow\|X\|_{\mathscr{F}}<+\infty \text {. }
$$

In a slightly different direction, let us mention that the problem of continuity and boundedness of convolution processes (1.1) with $Z$ a semi-martingale and $f(0)=0$ was addressed in [10], under general conditions. The following lemma yields a criterion ensuring (3.5) for $L_{p}$ and Hölder norms, under condition (3.3):

Lemma 3.5. Let $\|$.$\| be an L_{p}$-norm for some $1 \leq p \leq+\infty$ or a $\eta$-Hölder norm for some $0 \leq \eta<1$. There exists a constant $C$ independent of $n$ such that a.s.

$$
\left\|R^{H+n}\right\| \leq C n^{H-1 / \alpha}\left\|R^{H}\right\|
$$

for all $n \geq 1$.

Proof. Suppose first that $\|\cdot\|$ is an $L_{p}$-norm for some $1 \leq p<+\infty$. From Lemma 3.3 with $\beta=n$ and Stirling's formula, we get

$$
\left\|R^{H+n}\right\|_{p} \leq C n^{H-1 / \alpha+1}\left(\int_{0}^{1}\left|\int_{0}^{t}(t-s)^{n-1} R_{s}^{H} \mathrm{~d} s\right|^{p} \mathrm{~d} t\right)^{1 / p}
$$


for some constant $C$ independent of $n$. Then, Hölder's inequality entails

$$
\left\|R^{H+n}\right\|_{p} \leq C\left\|R^{H}\right\|_{p} n^{H-1 / \alpha+1}\left(\int_{0}^{1}\left|\int_{0}^{t} u^{q(n-1)} \mathrm{d} s\right|^{p / q} \mathrm{~d} t\right)^{1 / p}
$$

where $q$ is such that $1 / p+1 / q=1$. The desired estimate follows now easily, and the considerations for $p=+\infty$ are identical.

Suppose now that $\|$.$\| is an \eta$-Hölder norm for some $0<\eta<1$. Cutting the integral in two, changing variables, and using again Lemma 3.3 together with Stirling's formula, we get for every $0 \leq s<t \leq 1$

$$
\left|R_{t}^{H+n}-R_{s}^{H+n}\right| \leq C n^{H-1 / \alpha+1}\left|\int_{0}^{s}(s-u)^{n-1}\left(R_{u+v}^{H}-R_{u}^{H}\right) \mathrm{d} u+\int_{0}^{v}(t-u)^{n-1} R_{u}^{H} \mathrm{~d} u\right|
$$

with the notation $v=t-s$. This entails

$$
\begin{aligned}
\frac{\left|R_{t}^{H+n}-R_{s}^{H+n}\right|}{|t-s|^{\eta}} & \leq C n^{H-1 / \alpha+1}\left\|R^{H}\right\|_{\eta}\left|\int_{0}^{s}(s-u)^{n-1} \mathrm{~d} u+\int_{0}^{v} \frac{(t-u)^{n-1} u^{\eta}}{v^{\eta}} \mathrm{d} u\right| \\
& \leq C n^{H-1 / \alpha}\left\|R^{H}\right\|_{\eta}\left(s^{n}+t^{n}\right) \\
& \leq C n^{H-1 / \alpha}\left\|R^{H}\right\|_{\eta}
\end{aligned}
$$

as desired.

Remark 3.6. It would be quite interesting to see if this lemma remains true in a general Banach space $\mathscr{F}$ endowed, for example, with a $(\beta, p)$-semi-norm as defined in Section 2. Notice from (3.4) that this would be a direct consequence of the following estimate

$$
\left\|\mathscr{R}_{\alpha}: \mathscr{F} \rightarrow \mathscr{F}\right\| \leq \frac{C}{\Gamma(\alpha+1)}
$$

for a constant $C$ independent of $\alpha$. The authors could not find a general result of this kind in the literature, but it seems plausible that (3.6) holds for all $(\beta, p)$-lower semi-norms (i.e. not necessarily $p$-subadditive). For example, it is folklore that (3.6) is true in $L_{p}$-spaces, and we will give an argument for the sake of completeness: if $f \in L_{p}, 1 \leq p<+\infty$, and $q$ is such that $1 / p+1 / q=1$, then it follows from Hölder's inequality that

$$
\begin{aligned}
\left(\Gamma(\alpha)\left\|\mathscr{R}_{\alpha} f\right\|_{p}\right)^{p} & =\int_{0}^{1}\left|\int_{0}^{x}(x-t)^{\frac{\alpha-1}{q}}(x-t)^{\frac{\alpha-1}{p}} f(t) \mathrm{d} t\right|^{p} \mathrm{~d} x \\
& \leq \int_{0}^{1}\left(\int_{0}^{x}(x-t)^{\alpha-1} \mathrm{~d} t\right)^{p / q} \int_{0}^{x}(x-t)^{\alpha-1}|f(t)|^{p} \mathrm{~d} t \mathrm{~d} x \\
& \leq \alpha^{-p / q} \int_{0}^{1} \int_{0}^{1} \mathbf{1}_{\{t \leq x\}}(x-t)^{\alpha-1}|f(t)|^{p} \mathrm{~d} x \mathrm{~d} t \\
& =\alpha^{-p / q} \int_{0}^{1} \int_{t}^{1}(x-t)^{\alpha-1} \mathrm{~d} x|f(t)|^{p} \mathrm{~d} t \\
& =\alpha^{-p / q} \int_{0}^{1} \frac{(1-t)^{\alpha}}{\alpha}|f(t)|^{p} \mathrm{~d} t \leq \alpha^{-p / q-1}\|f\|_{p}^{p}=\alpha^{-p}\|f\|_{p}^{p}
\end{aligned}
$$

so that

$$
\left\|\mathscr{R}_{\alpha}: L_{p} \rightarrow L_{p}\right\| \leq \frac{1}{\Gamma(\alpha+1)}
$$


Besides, taking $f \equiv 1$ entails that (3.7) is indeed an equality. The same holds for $p=+\infty$ as well, as can be seen in a straightforward way. Using the same argument as in Lemma 3.5, we can also prove - details are left to the reader - that

$$
\left\|\mathscr{R}_{\alpha}: \mathscr{H}^{\eta} \rightarrow \mathscr{H}^{\eta}\right\|=\frac{1}{\Gamma(\alpha+1)},
$$

where $\mathscr{H}^{\eta}$ stands for the set of $\eta$-Hölderian functions starting from zero. Notice finally that if (3.6) held true as an equality, this would practically mean that the condition (3.3) is also necessary to ensure (3.5).

We can now finish the proof of the lower bound in Theorem 3.1, under the sole assumption (3.3):

Corollary 3.7. Let $X$ be defined by (1.2) with $0<\alpha<2, H \geq 1 / \alpha$, and such that (3.3) holds. For all $1 \leq p \leq+\infty$, there exists $C>0$ such that

$$
\log \mathbb{P}\left(\|X\|_{p} \leq \varepsilon\right) \geq-C \varepsilon^{-1 / H}, \quad \varepsilon \rightarrow 0 .
$$

Besides, for all $0<\eta<1$,

$$
\log \mathbb{P}\left(\|X\|_{\eta} \leq \varepsilon\right) \geq-C \varepsilon^{-1 /(H-\eta)}, \quad \varepsilon \rightarrow 0
$$

for some constant $C>0$, as soon as $H>1 / \alpha+\eta$.

Proof. Consider first the $L_{p}$-norms. By Lemma 3.5 and the triangle inequality, one has

$$
\|X\|_{p} \leq \sum_{n=0}^{\infty}\left|a_{n}\right|\left\|R^{H+n}\right\|_{p} \leq\left(1+C \sum_{n=1}^{\infty}\left|a_{n}\right| n^{H-1 / \alpha}\right)\left\|R^{H}\right\|_{p} \leq C\left\|R^{H}\right\|_{p}
$$

where the last inequality comes from condition (3.3). Hence,

$$
\log \mathbb{P}\left(\|X\|_{p} \leq \varepsilon\right) \geq \log \mathbb{P}\left(\left\|R^{H}\right\|_{p} \leq \varepsilon / C\right) \geq-C \varepsilon^{-1 / H}
$$

where the last inequality comes form the results proved (in the continuous case $H>1 / \alpha$ ) or mentioned (in the discontinuous case $H=1 / \alpha$ ) in [16]. The proof is exactly the same for the $\eta$-Hölder norms, using Theorem 7 in [16].

Remark 3.8. It follows from the above proof and Theorem 7 in [16] that if (3.6) were true, then Corollary 3.7 would also hold for all $(\beta, p)$-lower semi-norms, with the formula for the rate given by Theorem 2.1.

\subsection{The upper bound for $L_{p}$-norms}

Our arguments for the upper bound will be more involved than for the lower bound, because of the nonindependence of the summands which makes the decomposition (1.2) less tractable. In particular, it seems difficult to find a method relying on Anderson's inequality, as in [16]. Notice first that in the case of $L_{p}$-norms it will be sufficient to prove the upper bound for $L_{1}$ only, in view of the obvious inequality $\|\cdot\|_{1} \leq\|\cdot\|_{p}$.

As mentioned in the introduction, we will use the general relation between small deviations for stable processes and entropy numbers for stable operators discovered by Li and Linde [15]. Set $E=L_{\infty}$ and consider the dual Banach space $E^{\prime}$ of $E$. It is known that $L_{1}$ is continuously embedded in $E^{\prime}$ with $\|f\|_{1}=\|f\|_{E^{\prime}}$, for all $f \in L_{1}$. For every $n \geq 0$, it is easily seen that the operator $\mathscr{R}_{n}^{H}: E \rightarrow L_{\alpha}$ defined by

$$
\mathscr{R}_{n}^{H}=\Gamma(H-1 / \alpha+n+1) \mathscr{R}_{H-1 / \alpha+n+1}
$$

where $\mathscr{R}_{H-1 / \alpha+n+1}$ is the Riemann-Liouville operator from Definition 3.4, is a linear operator such that

$$
\left\|\mathscr{R}_{n}^{H}: E \rightarrow L_{\alpha}\right\|=1 .
$$


Besides, $\mathscr{R}_{n}^{H}$ generates the Riemann-Liouville process $R^{H+n}$ in the sense of [15] since, by Theorem 11.4.1 in [19],

$$
\mathbb{E}\left[\exp \left(i \int_{0}^{1} R_{t}^{H+n} x(t) \mathrm{d} t\right)\right]=\exp \left(-\left\|\mathscr{R}_{n}^{H} x\right\|_{\alpha}^{\alpha}\right) \quad \text { for every } x \in E .
$$

Using (3.3), Stirling's formula and Proposition 3.5.1 in [19], this entails that the operator $\mathscr{R}_{f}: E \rightarrow L_{\alpha}$ defined by

$$
\mathscr{R}_{f}=\sum_{n \geq 0} a_{n} \mathscr{R}_{n}^{H}
$$

is a bounded linear operator generating $X$ in the sense of [15]. Recall now the definition of the dyadic entropy numbers of an operator $u: E \rightarrow F$, where $F$ is some other quasi-Banach space:

$$
e_{k}[u: E \rightarrow F]=\inf \left\{\varepsilon>0 \mid \exists y_{1} \ldots y_{2^{k-1}} \in F: u\left(B_{E}(0,1)\right) \subseteq \bigcup_{i=1}^{2^{k-1}} B_{F}\left(y_{i}, \varepsilon\right)\right\}
$$

for every $k \geq 1$, where $B_{E}(x, \varepsilon)$ denotes the ball in $E$ with centre $x$ and radius $\varepsilon$ and with the same notation for $B_{F}\left(y_{i}, \varepsilon\right)$. From Theorem 4.5 in Li-Linde [15] - see also [1] for a general result, the first one to our knowledge, ensuring that $E^{\prime}$ satisfies property D in [15] - our upper bound will be proved as soon as we can show that there exists a constant $C>0$ such that

$$
e_{k}\left[\mathscr{R}_{f}: E \rightarrow L_{\alpha}\right] \geq C k^{-(H-1 / \alpha-1)}, \quad k \rightarrow \infty .
$$

To find this estimate, we will need a lemma on the entropy numbers of RL operators:

Lemma 3.9. For all $\beta>1 / 2$ and $0<\alpha \leq 2$, one has

$$
e_{k}\left[\mathscr{R}_{\beta}: E \rightarrow L_{\alpha}\right] \approx k^{-\beta}, \quad k \rightarrow \infty .
$$

Proof. From the main result of [16], we know that for every $H>0$

$$
-\log \mathbb{P}\left(\left\|R^{H}\right\|_{E^{\prime}} \leq \varepsilon\right)=-\log \mathbb{P}\left(\left\|R^{H}\right\|_{1} \leq \varepsilon\right) \approx \varepsilon^{-1 / H}, \quad \varepsilon \rightarrow 0 .
$$

Suppose first that $\alpha=2$. It follows from the general Gaussian connection established in [9] and [14] that for every $\beta>1 / 2$

$$
e_{k}\left[\mathscr{R}_{\beta}: E \rightarrow L_{2}\right] \approx k^{-\beta}, \quad k \rightarrow \infty,
$$

which completes the proof for $\alpha=2$, and yields the upper bound for all $\alpha \leq 2$ because $L_{2} \subseteq L_{\alpha}$. To obtain the lower bound we first make the obvious remark that

$$
e_{k}\left[\mathscr{R}_{\beta}: E \rightarrow L_{\alpha}\right] \geq e_{k}\left[\mathscr{R}_{\beta}: \mathscr{C} \rightarrow L_{\alpha}\right]
$$

where $\mathscr{C} \subseteq E$ stands for the set of continuous functions from $[0,1]$ to $\mathbb{R}$. On the other hand, from interpolation results for entropy numbers - see e.g. the Theorem p. 13 in [6] - there exists $C>0$ independent of $k$ such that

$$
e_{k}\left[\mathscr{R}_{\beta}: \mathscr{C} \rightarrow \mathscr{C}\right]^{1-\alpha / 2} e_{k}\left[\mathscr{R}_{\beta}: \mathscr{C} \rightarrow L_{\alpha}\right]^{\alpha / 2} \geq C e_{2 k}\left[\mathscr{R}_{\beta}: \mathscr{C} \rightarrow L_{2}\right]
$$

But it follows from Proposition 6.1 in [14] that

$$
e_{k}\left[\mathscr{R}_{\beta}: \mathscr{C} \rightarrow L_{2}\right] \approx k^{-\beta} \text { and } e_{k}\left[\mathscr{R}_{\beta}: \mathscr{C} \rightarrow \mathscr{C}\right] \approx k^{-\beta}
$$

as $k \rightarrow \infty$. Putting these last two facts together yields the desired lower bound. 
End of the proof. Define $h(x)=f(x)-x^{H-1 / \alpha}$. With the above notation, we have

$$
\mathscr{R}_{h}=\sum_{n \geq 1} a_{n} \mathscr{R}_{n}^{H}=\mathscr{R}_{f}-\mathscr{R}_{H-1 / \alpha+1} .
$$

By Lemma 3.9 and the additivity property of entropy numbers, this entails

$$
e_{k}\left[\mathscr{R}_{f}: E \rightarrow L_{\alpha}\right]+e_{k}\left[\mathscr{R}_{h}: E \rightarrow L_{\alpha}\right] \geq e_{2 k+1}\left[\mathscr{R}_{H-1 / \alpha+1}: E \rightarrow L_{\alpha}\right] \approx k^{-(H-1 / \alpha+1)}
$$

as $k \rightarrow \infty$. Fix $0<\delta<1$ such that (3.2) holds. By the triangle inequality and the semi-group property of the Riemann-Liouville operator $\left(\mathscr{R}_{\beta} \circ \mathscr{R}_{\gamma}=\mathscr{R}_{\beta+\gamma}\right.$ for all $\left.\beta, \gamma>0\right)$ we see that for every $x \in E$

$$
\begin{aligned}
\left\|\mathscr{R}_{h} x\right\|_{2} & \leq \sum_{n=1}^{\infty}\left|a_{n}\right| \Gamma(H-1 / \alpha+n+1)\left\|\mathscr{R}_{n-\delta}: L_{2} \rightarrow L_{2}\right\|\left\|\mathscr{R}_{H-1 / \alpha+1+\delta} x\right\|_{2} \\
& \leq\left\|\mathscr{R}_{H-1 / \alpha+1+\delta} x\right\|_{2} \sum_{n=1}^{\infty}\left|a_{n}\right| \frac{\Gamma(H-1 / \alpha+n+1)}{\Gamma(n+1-\delta)} \\
& \leq C\left\|\mathscr{R}_{H-1 / \alpha+1+\delta} x\right\|_{2} \sum_{n=1}^{\infty}\left|a_{n}\right| n^{H-1 / \alpha+\delta}
\end{aligned}
$$

where the second line comes from (3.7). By assumption (3.2), we get

$$
\left\|\mathscr{R}_{h} x\right\|_{2} \leq C\left\|\mathscr{R}_{H-1 / \alpha+1+\delta} x\right\|_{2}
$$

for all $x \in E$, and it follows from Lemma 4.2 in [?] that

$$
e_{k}\left[\mathscr{R}_{h}: E \rightarrow L_{2}\right] \leq C e_{k}\left[\mathscr{R}_{H-1 / \alpha+1+\delta}: E \rightarrow L_{2}\right] \approx k^{-(H-1 / \alpha+1+\delta)},
$$

the last approximation coming from Lemma 3.9. Recalling the obvious inequality

$$
e_{k}\left[\mathscr{R}_{h}: E \rightarrow L_{\alpha}\right] \leq e_{k}\left[\mathscr{R}_{h}: E \rightarrow L_{2}\right]
$$

for every $\alpha \leq 2$ and putting this together with (3.9) yields

$$
e_{k}\left[\mathscr{R}_{f}: E \rightarrow L_{\alpha}\right] \geq C k^{-(H-1 / \alpha+1)}-e_{k}\left[\mathscr{R}_{h}: E \rightarrow L_{\alpha}\right] \geq C k^{-(H-1 / \alpha+1)}
$$

for large enough $k$, which completes the proof.

Remark 3.10. Using additivity of entropy numbers, one can show that

$$
e_{k}\left[\mathscr{R}_{f}: E \rightarrow L_{\alpha}\right] \leq C k^{-(H-1 / \alpha+1)}
$$

for some $C>0$ and large enough $k$, so that actually

$$
e_{k}\left[\mathscr{R}_{f}: E \rightarrow L_{\alpha}\right] \approx k^{-(H-1 / \alpha+1)}
$$

when $k \rightarrow \infty$. See also [2] for a more general connection between lower estimates of the small deviation probabilities and upper estimates of entropy numbers, completing the results of [15]. 


\subsection{The upper bound for $\eta$-Hölder norms}

This will be a rather easy consequence of the above upper bound for $L_{\infty}$-norms, and we shall not use entropy numbers anymore. A direct approach using dual Hölder spaces [6] may provide more precise results, but it promises to be much more involved as far as the entropy numbers are concerned. Fix $\eta \in(0,1)$ and $H>1 / \alpha+\eta$. Consider the process $Y^{\nu}$ defined by

$$
Y_{t}^{\nu}=\sum_{n \geq 0} a_{n}\left(\frac{\Gamma(H+n-1 / \alpha+1)}{\Gamma(H-\nu+n-1 / \alpha+1)}\right) R_{t}^{H-\nu+n}
$$

for some $0<\nu<\eta$. Since $H>1 / \alpha+\nu$ we know that $R^{H-\nu+n} \in \mathscr{C}$ a.s. Using assumption (3.3) and reasoning exactly as in Lemma 3.5, we see that the sum (3.10) converges uniformly and $Y^{\nu} \in \mathscr{C}$ by Dini's lemma Lemma 3.3 and the uniform convergence in (3.10) that

$$
X=\mathscr{R}_{\nu} Y^{\nu}
$$

The inverse of the Riemann-Liouville operator $\mathscr{R}_{\nu}$ is the so-called fractional differentiation operator $\mathscr{D}_{\nu}$ defined by

$$
\mathscr{D}_{\nu}=\mathscr{D} \circ \mathscr{R}_{1-\nu}
$$

where $\mathscr{D}$ is the standard differentiation operator [7]. For every $\gamma \in[0,1)$ we set, as above, $\mathscr{H}^{\gamma}$ for the set of $\gamma$-Hölderian functions $[0,1] \rightarrow \mathbb{R}$ starting from 0 , endowed with the norm $\|\cdot\|_{\gamma}$. It follows from Theorem 14 in [7] that for any $\gamma>0$ such that $\nu+\gamma<1$,

$$
\mathscr{R}_{\nu}: \mathscr{H}^{\gamma} \rightarrow \mathscr{H}^{\nu+\gamma}
$$

is bounded and bijective. By the open mapping theorem, the same holds for

$$
\mathscr{D}_{\nu}: \mathscr{H}^{\nu+\gamma} \rightarrow \mathscr{H}^{\gamma}
$$

Set $\gamma=\eta-\nu$. The assumption $H>1 / \alpha+\eta$ ensures that $X \in \mathscr{H}^{\eta}$, so that a.s. $Y^{\nu} \in \mathscr{H}^{\gamma}$ and

$$
\left\|Y^{\nu}\right\|_{\infty} \leq\left\|Y^{\nu}\right\|_{\gamma} \leq\left\|\mathscr{D}_{\nu}: \mathscr{H}^{\eta} \rightarrow \mathscr{H}^{\gamma}\right\|\|X\|_{\eta}
$$

By (3.10), (3.2) and the upper bound for $L_{p}$-norms, we obtain

$$
\log \mathbb{P}\left[\|X\|_{\eta}<\varepsilon\right] \leq \log \mathbb{P}\left[\left\|Y^{\nu}\right\|_{\infty}<K \varepsilon\right] \leq-C \varepsilon^{-1 /(H-\nu)}, \quad \varepsilon \rightarrow 0
$$

for some constants $C, K>0$. This completes the proof in letting $\nu \rightarrow \eta$.

Remark 3.11. If the operator

$$
\mathscr{D}_{\nu}: \mathscr{H}^{\nu} \rightarrow \mathscr{C}
$$

were continuous for the Hölder topology, then the above simple argument would readily yield the exact upper bound

$$
\log \mathbb{P}\left[\|X\|_{\eta}<\varepsilon\right] \leq-C \varepsilon^{-1 /(H-\eta)}, \quad \varepsilon \rightarrow 0
$$




\section{Two EXAmples}

\subsection{Stable Ornstein-Uhlenbeck processes}

These processes solve the Langevin equation

$$
X_{t}=\xi-\lambda \int_{0}^{t} X_{s} \mathrm{~d} s+\sigma Z_{t}
$$

where $\lambda, \xi, \sigma \in \mathbb{R}$ and $Z$ is a symmetric $\alpha$-stable Lévy process. The equation has a unique explicit solution given by

$$
X_{t}=\mathrm{e}^{-\lambda t}\left(\xi+\sigma \int_{0}^{t} \mathrm{e}^{\lambda u} \mathrm{~d} Z_{u}\right)
$$

and since in this paper we focus on processes starting from zero, our stable OU process takes here the simple form:

$$
X_{t}=\sigma \int_{0}^{t} \mathrm{e}^{-\lambda(t-u)} \mathrm{d} Z_{u}
$$

In the non-Gaussian case, the process $X$ has jumps and we can consider $L_{p}$-norms only. Since the kernel $g(t)=\sigma \mathrm{e}^{-\lambda t}$ satisfies (3.2), Theorem 3.1 entails

$$
\log \mathbb{P}\left(\|X\|_{p} \leq \varepsilon\right) \approx-\varepsilon^{-\alpha}, \quad \varepsilon \rightarrow 0
$$

In the Gaussian case the process $X$ has the same regularity as the Brownian motion and Theorem 2.1 entails that its small deviation probabilities are also exactly the same, up to the normalization given by $\sigma$. We refer $e . g$. to Section 6 in [16] for a survey on small deviations of Brownian motion under different norms. In particular, one has

$$
\varepsilon^{2} \log \mathbb{P}\left(\|X\|_{\infty} \leq \varepsilon\right) \rightarrow-\frac{\pi^{2}}{8} \sigma^{2}, \quad \varepsilon \rightarrow 0 .
$$

This is actually a very particular case of a general result due to Berthet and Shi [3], who studied small deviations under the sup norm for Gaussian Markov processes defined by the generalised Lamperti transformation

$$
X_{t}=h(t) B_{g(t)}
$$

of a linear Brownian motion (in the OU case, one has $g(t)=\mathrm{e}^{2 \lambda t}-1$ and $h(t)=\sigma \mathrm{e}^{-\lambda t} / \sqrt{2|\lambda|}$ ), as a consequence of small ball results for the Brownian motion under a weighted sup-norm. Notice that small deviations of Gaussian Markov processes under $L_{p}$ norms were also thoroughly studied in [12]. However, there does not seem to exist any literature for this class of processes under other norms.

It is natural to ask whether the constant exists in the asymptotic (4.2) in the non-Gaussian case. Unfortunately, the absence of self-similarity prevents us from using any subadditivity argument as in [4] or [16]. Besides, notice that non-Gaussian stable OU processes cannot be recovered from a Lévy stable process by a Lamperti transformation.

\subsection{Fractional Ornstein-Uhlenbeck processes}

Let us consider the Gaussian case first. These processes were introduced in [5] as the solution to the generalised Langevin equation

$$
X_{t}=\xi-\lambda \int_{0}^{t} X_{s} \mathrm{~d} s+\sigma B_{t}^{H}
$$

driven by a fractional Brownian motion $B^{H}$ with Hurst parameter $H$. They can be written similarly as

$$
X_{t}=\mathrm{e}^{-\lambda t}\left(\xi+\sigma \int_{0}^{t} \mathrm{e}^{\lambda u} \mathrm{~d} B_{u}^{H}\right)
$$


(in [5] Prop. A.1 it is proved that the above integral with respect to $B^{H}$ exists as a Riemann-Stieltjes integral). In [5], it is also remarked that except in the Brownian case, $X$ defined in (4.3) cannot be retrieved from a fBM by the Lamperti transformation. As above, we will consider the case $\xi=0$ viz.

$$
X_{t}=\sigma \int_{0}^{t} \mathrm{e}^{-\lambda(t-u)} \mathrm{d} B_{u}^{H}, \quad t \in[0,1]
$$

It is well-known that $B^{H}$ can be decomposed into

$$
B_{t}^{H}=c_{H}\left(R_{t}^{H}+M_{t}^{H}\right)
$$

where $c_{H}$ is a normalization constant, $R^{H}$ the above (Gaussian) Riemann-Liouville process and $M^{H}$ an independent smooth process. Using the same argument as in [16], Theorem 12, and the independence argument relying on Anderson's inequality detailed in [13], p. 1334, we see that $X$ defined in (4.4) has exactly the same small deviation probabilities as $Y$ defined by

$$
Y_{t}=\sigma c_{H} \int_{0}^{t} \mathrm{e}^{-\lambda(t-u)} \mathrm{d} R_{u}^{H}=\sigma c_{H} \int_{0}^{t} \mathrm{e}^{-\lambda(t-u)}(t-u)^{H-1 / 2} \mathrm{~d} B_{u}, \quad t \in[0,1] .
$$

From Theorem 2.1 we deduce that for any seminorm $\|\cdot\| \in \mathbf{N}(\beta, p)$ such that $H>\beta+1 / p$, we have

$$
\lim _{\varepsilon \downarrow 0} \varepsilon^{\gamma} \log \mathbb{P}[\|X\| \leq \varepsilon]=-\mathscr{K}\left(\sigma c_{H}\right)^{\gamma}
$$

where $\gamma=(H-\beta-1 / p)^{-1}$ and $\mathscr{K}$ is given by $(2.1)$.

In [17], a stable extension of (4.3) is proposed, replacing the driving fractional Brownian motion by the so-called linear fractional stable motion $\Delta_{H, \alpha}^{a, b}$ (LFSM) which is defined by

$$
\Delta_{H, \alpha}^{a, b}(t)=\int_{\mathbb{R}}\left(a\left[(t-s)_{+}^{H-1 / \alpha}-(-s)_{+}^{H-1 / \alpha}\right]+b\left[(t-s)_{-}^{H-1 / \alpha}-(-s)_{-}^{H-1 / \alpha}\right]\right) \mathrm{d} Z_{s}
$$

for every $t \geq 0$, where $a, b$ are two real constants, $0<\alpha \leq 2$ and $0<H<1$. A remarkable feature of these LFSM's is that they are all equal to fBM - up to some constant - in the case $\alpha=2$. In the non-Gaussian case however, different values of $a, b$ yield different processes. Taking $a=1$ and $b=0$ entails

$$
\Delta_{H, \alpha}^{1,0}(t)=\int_{\mathbb{R}}\left((t-s)_{+}^{H-1 / \alpha}-(-s)_{+}^{H-1 / \alpha}\right) \mathrm{d} Z_{s}
$$

we can decompose

$$
\Delta_{H, \alpha}^{1,0}(t) \stackrel{d}{=} R_{t}^{H}+M_{t}^{H}
$$

where $R^{H}$ is the above (non-Gaussian) Riemann-Liouville process and $M^{H}$ given by

$$
M_{t}^{H}=\int_{0}^{+\infty}\left((t+s)^{H-1 / \alpha}-s^{H-1 / \alpha}\right) \mathrm{d} Z_{s}, \quad t \geq 0
$$

is an independent smooth process. The solution to (4.3) starting from 0 can also be written as

$$
X_{t}=\sigma \int_{0}^{t} \mathrm{e}^{-\lambda(t-u)} \mathrm{d} \Delta_{H, \alpha}^{1,0}(u), \quad t \in[0,1]
$$


We refer to [21] for much more on these processes with a view towards applications. Reasoning as above, we see that their small deviations are the same as those of

$$
Y_{t}=\sigma \int_{0}^{t} \mathrm{e}^{-\lambda(t-u)} \mathrm{d} R_{u}^{H}=\sigma \int_{0}^{t} \mathrm{e}^{-\lambda(t-u)}(t-u)^{H-1 / \alpha} \mathrm{d} Z_{u}, \quad t \in[0,1] .
$$

As mentioned in [17] Proposition 3.3, the problem is only relevant for $1 / \alpha \leq H<1$ and $1<\alpha<2$. The case $H=1 / \alpha$ was studied in the last paragraph. In the case $H>1 / \alpha$ we can apply Theorem 3.1 as well, and an interesting feature is that we can include some Hölder norms: one has

$$
\log \mathbb{P}\left(\|X\|_{\eta} \leq \varepsilon\right)=-\varepsilon^{-1 /(H-\eta)+o(1)}, \quad \varepsilon \rightarrow 0
$$

as soon as $H>1 / \alpha+\eta$.

Acknowledgements. This work was carried out within a French-German project Procope, whose support is gratefully acknowledged.

\section{REFERENCES}

[1] S. Artstein, V. Milman and S.J. Szarek, Duality of metric entropy. Ann. Math. (2) 159 (2004) 1213-1328.

[2] F. Aurzada., Metric entropy and the small deviation problem for stable processes. To appear in Prob. Math. Stat. (2006).

[3] P. Berthet and Z. Shi, Small ball estimates for Brownian motion under a weighted sup-norm. Studia Sci. Math. Hungar. 36 (2000) 275-289.

[4] J. Bertoin, On the first exit time of a completely asymmetric stable process from a finite interval. Bull. London Math. Soc. 28 (1996) 514-520.

[5] P. Cheridito, H. Kawaguchi and M. Maejima, Fractional Ornstein-Uhlenbeck Processes. Elec. J. Probab. 8 (2003) 1-14.

[6] D.E. Edmunds and H. Triebel, Function spaces, entropy numbers, differential operators. Cambridge University Press (1996).

[7] G.H. Hardy and J.E. Littlewood, Some properties of fractional integrals I. Math. Z. 27 (1927) 565-606.

[8] D. Heath, R.A. Jarrow and A. Morton, Bond pricing and the term structure of interest rate: A new methodology for contingent claim valuation. Econometrica 60 (1992) 77-105.

[9] J. Kuelbs and W.V. Li, Metric Entropy and the Small Ball Problem for Gaussian Measures. J. Func. Anal. 116 (1993) $113-157$.

[10] S. Kwapien, M.B. Marcus and J. Rosiński, Two results on continuity and boundedness of stochastic convolutions. Ann. Inst. Henri Poincaré, Probab. et Stat. 42 (2006) 553-566.

[11] W.V. Li, A Gaussian correlation inequality and its application to small ball probabilities. Elec. Comm. Probab. 4 (1999) $111-118$.

[12] W.V. Li, Small ball probabilities for Gaussian Markov processes under the $L_{p}$-norm. Stochastic Processes Appl. 92 (2001) $87-102$.

[13] W.V. Li and W. Linde, Existence of small ball constants for fractional Brownian motions. C. R. Acad. Sci. Paris 326 (1998) $1329-1334$

[14] W.V. Li and W. Linde, Approximation, metric entropy and the small ball problem for Gaussian measures. Ann. Probab. 27 (1999) 1556-1578.

[15] W.V. Li and W. Linde, Small Deviations of Stable Processes via Metric Entropy. J. Theoret. Probab. 17 (2004) $261-284$.

[16] M.A. Lifshits and T. Simon, Small deviations for fractional stable processes. Ann. Inst. H. Poincaré Probab. Statist. 41 (2005) $725-752$.

[17] M. Maejima and K. Yamamoto, Long-Memory Stable Ornstein-Uhlenbeck Processes. Elec. J. Probab. 8 (2003) 1-18.

[18] S.G. Samko, A.A. Kilbas and O.I. Marichev, Fractional Integrals and Derivatives. Gordon and Breach (1993).

[19] G. Samorodnitsky and M.S. Taqqu, Stable Non-Gaussian Random Processes. Chapman \& Hall (1994).

[20] K. Takashima, Sample path properties of ergodic self-similar processes. Osaka J. Math. 26 (1989) 159-189.

[21] M.S. Taqqu and R.L. Wolpert, Fractional Ornstein-Uhlenbeck Lévy Processes and the Telecom Process: Upstairs and Downstairs. Signal Processing 85 (2005) 1523-1545. 